\title{
A case report looking at ACE inhibitors as the cause of angioedema during dental treatment
}

IN BRIEF
- Identifies patients at risk of developing
angioedema.
- Highlights differences between
angioedema and anaphylaxis.
Presents the treatment of angioedema in
practice.

P. Raval ${ }^{1}$

Angiotensin converting enzymes, also known as ACE inhibitors are regularly prescribed by doctors in the treatment of congestive heart failure and hypertension. In this case report, we will be looking at the delayed onset of angioedema as a result of ACE inhibitors that occurred during dental treatment. We will discuss other causes of angioedema and its management and aim to raise awareness among clinicians to include this in their differential diagnosis of swellings around the mouth, particularly as it can mimic the initial response or be a prelude to an anaphylactic shock.

\section{INTRODUCTION}

Swellings in and around the mouth are of great importance to dentists who should have a thorough understanding of them and be able to provide a differential diagnosis even when their cause(s) may not always be obvious.

Angioedema is a rapid and reactive type of swelling that can be potentially life threatening. It usually occurs around the lips, tongue and mouth involving the mucosal and sub-mucosal layers. There are different causes of angioedema including allergic, hereditary, idiopathic and drug induced angioedemas. ${ }^{1}$ Drug-induced angioedemas are diagnosed when no other clinical cause of angioedema can be found and no future episodes recur following cessation of the drug.

One cause of drug-induced angioedemas is angiotensin converting enzymes (ACE) inhibitors, a commonly prescribed medicine regularly seen in general practice. It is estimated that 35-40 million patients take this medicine worldwide. ${ }^{2}$ This association is well established and can be life threatening, especially when involving the mucosal and submucosal layers of the upper airway. ${ }^{3}$

ACE inhibitor induced angioedema is a rare side effect and figures range from $0.1 \%$ to $0.2 \%$ of patients being affected worldwide. ${ }^{4}$

Clinical teacher at Kings College London and general dental practitioner, 6 Tangmere Gardens, Northolt, Middlesex, UB5 6LP

Correspondence to: Pritesh Raval

Email: pritesh.raval@sky.com; Tel: 07852188017

\section{Refereed Paper}

Accepted 25 September 2013

DOI: 10.1038/sj.bdj.2014.2

${ }^{\circ}$ British Dental Journal 2014; 216: 73-75
There is not a clear relationship between commencing the medication and the onset of angioedema. In 25\% of cases of angioedema secondary to ACE inhibitors, the first episode of angioedema will usually occur in the first month of starting ACE inhibitor therapy. However, it is not uncommon for a patient to be taking ACE inhibitors for many years before any side effects become apparent. ${ }^{5,6}$

In a case reported by Kozel et al., a 49-yearold Afro-Caribbean patient had been taking ACE inhibitor medication for over four years when he started intermittently developing a swelling around the tongue, lips and oropharynx, which increased in frequency and severity until he was diagnosed with angioedema secondary to ACE inhibitors. He ceased taking the medication and these episodes resolved.

Angioedema related to ACE inhibitor therapy does not appear to be dose related and the time course of resolution can range from hours to several days. ${ }^{8}$ Any area of the body may develop angioedema (with or without urticaria), including the gastrointestinal (GI) tract,, ${ }^{1,9}$ however, it is more common in the head and neck region (Fig. 1). ${ }^{1,6}$ The common sites associated with ACE inhibitor medication are the tongue, oropharynx, perioral and periorbital regions. It is not completely understood why, but this complication of ACE inhibitors is three times more common among the Afro-Caribbean population. ${ }^{6,10}$

\section{CASE REPORT}

A 77-year-old Afro-Caribbean male undergoing dental treatment in a general practice started developing a progressive swelling involving his lips, tongue and buccal mucosa. The swelling started to spread rapidly (Fig. 2). Initially it appeared to be a type I allergic reaction. All treatment was immediately stopped and extra assistance was called. The emergency drugs trolley was deployed and the patient was continually reassured while the medical history was rechecked. There were no known allergies and no history of swellings.

The patient suffered from type II diabetes and hypertension, both of which were well controlled with medication. His medication list comprised of Metformin, Amlodipine, Atenolol, Ramipril and Allopurinol. Apart from this, the medical history was unremarkable.

Similar composite fillings had been conducted a week prior and the treatment was uneventful. Adrenaline and an antihistamine were administered to the patient and an ambulance was called to take him to the hospital.

At the accident and emergency department, the patient was continued on adrenaline and corticosteroids and his history was thoroughly checked. Blood tests were conducted and all baseline records were continuously recorded and monitored. After a couple of hours the swelling started to subside. He was diagnosed as having angioedema secondary to ACE inhibitors, which he had been taking for over four years.

A report was immediately sent back to his general medical practitioner asking him to investigate and if necessary, change his ACE inhibitor medication. An annual follow up was conducted and there had been no further reports of any forms of angioedema from the patient since the change to his medication. 


\section{DISCUSSION}

Angioedema secondary to ACE inhibitors is a serious complication and some deaths have been reported. ${ }^{11-13}$ There are a few selected cases in the literature of angioedema developing and presenting in the dental setting, ${ }^{14}$ which reports a wellestablished link between angioedema and ACE inhibitors. ${ }^{4}$

Angioedema is a much less common side effect of ACE inhibitors compared to other side effects such as a non-productive cough that is seen in about $10 \%$ of patients treated with them. ${ }^{15}$ It is becoming increasingly more common for two reasons. The first is the effectiveness of these drugs in the treatment of congestive heart failure and hypertension and the second is due to the rise in life expectancy in western societies, which has led to more patients with hypertension and congestive heart failure being prescribed these drugs.

The National Institute for Health and Care Excellence advise that ACE inhibitors should be prescribed to people under the age of 55 who suffer from hypertension or an angiotensin II receptor blocker if the ACE inhibitor is not tolerable. If the patient is over 55 or of Afro-Caribbean origin then a calcium channel blocker and an ACE inhibitor should be prescribed. ${ }^{16}$

The swelling related to ACE inhibitor treatment can be severe and potentially life threatening and therefore must always be considered in the medical history as well as when investigating swellings around the head and neck region, especially if associated with the tongue, lips, neck and inside of the mouth. ${ }^{13}$

Swellings around the mouth and tongue region are quite often attributed to food allergies and so it is important to differentiate this from ACE inhibitor-related swellings. ${ }^{4}$

ACE inhibitors produce vasodilation by inhibiting the formation of angiotensin II. Angiotensin II is a potent vasoconstrictor formed by the proteolytic action of rennin (released by the kidneys) acting on circulating angiotensinogen to form angiotensin I. Angiotensin I is then converted to angiotensin II by angiotensin converting enzymes (in response to a fall in the blood pressure). The angiotensin II therefore helps increase the blood pressure by causing vasoconstriction and also increases smooth muscle hypertrophy; both of which can lead to an increase in blood pressure. The ACE inhibitor therefore prevents conversion of angiotensin I to angiotensin II thereby lowering arteriolar resistance and increasing venous capacity. ACE inhibitors decrease angiotensin II and aldosterone levels on the one hand and increase the level of bradykinin and prolong its action on the other. Bradykinin is a peptide that is a potent vasodilating substance, which helps reduce blood pressure. ACE inhibitors increase the level of bradykinin by preventing its degradation, further lowering blood pressure. However, the bradykinin is also responsible for causing the persistent dry cough seen as a side effect of the ACE inhibitors as well as the angioedema. These mechanisms can cause fluid extravasation into the subcutaneous tissues, which produces angioedema.

Afro-Caribbean people are more at risk as racial differences in Kallikrein-Kinin (a system of blood proteins that play a role in inflammation and coagulation) leaves patients of this ethic origin with an increased sensitivity to bradykinin. ${ }^{6}$

The first step when dealing with people with angioedema, particularly in the dental setting, is to eliminate other causes of rapid swellings around the head and neck region including anaphylaxis. This involves rechecking the medical history for any allergies and questioning the patient. The next step is to secure the patency of the upper airway by improving their positioning and providing oxygen. The medications in the emergency drugs box should be used such as adrenaline in severe cases of angioedema or antihistamines in less severe forms of angioedema; ${ }^{1}$ and if needed intubation and surgical intervention, which would be conducted by a trained specialist in the hospital setting. Patients should be carefully monitored as they usually have hypertension, heart disease and other illnesses, which can make administering adrenaline or other corticosteroids hazardous.

Long-term management includes changing the patient's medication to angiotensin II receptor antagonists as they are well tolerated and decrease cardiovascular mortality and morbidity. ${ }^{13}$ Angiotensin II receptor antagonists do not block bradykinin metabolism, however, reports have been published that suggest they may also cause angiodema. ${ }^{17}$ Generally, an interval of about six months is recommended to exclude the possibility of idiopathic or non-ACE inhibitor-related causes of angioedema. ${ }^{18}$

Other causes of angioedema include hereditary/congenital causes. This is due to an autosomal dominant condition whereby there is a C1 inhibitor deficiency leading to increased levels of bradykinin, resulting in angioedema because of leakage of fluid from blood vessels into connective tissue. Angioedema related to C1 inhibitor deficiency and ACE inhibitors is not responsive to antihistamines. ${ }^{19}$ In addition it must be stressed that angioedema secondary to ACE inhibitors is not the same as an

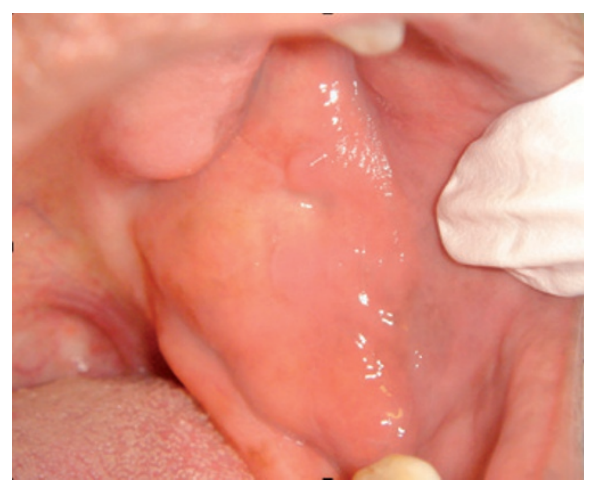

Fig. 1 A swelling developing around the buccal mucosa

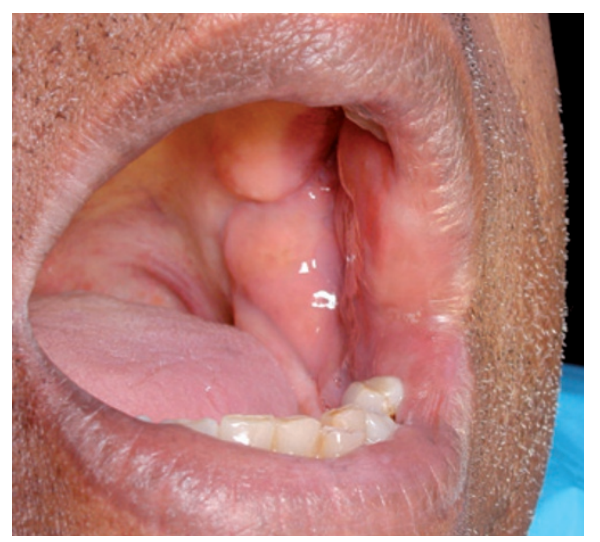

Fig. 2 A swelling around the lips, tongue and intraorally

allergic reaction that results in angioedema. ACE inhibitor-related angioedema has a much more prolonged half-life.

\section{CONCLUSION}

Angioedema of the head and neck is potentially a life threatening condition that needs urgent attention and treatment. This could quite easily present to the general dental practitioner who needs to be aware of causes of swellings around the head and neck region, in particular angioedema of the tongue, lips and buccal mucosa. It is prudent for dentists to be aware of the link between ACE inhibitors and angioedema and it should form part of the differential diagnosis before being referred to the relevant colleague for treatment.

1. Kanokvalai K, Sukhum J, Kanonrat B, Kowit J. Angioedema: clinical and etiological aspects. Clin Dev Immunol 2007; 26438.

2. Agostoni A, Cicardi M. Drug-induced angioedema without urticaria. Drug Safety 2001; 24: 599-606.

3. Kupfer Y, Ramachandran K, Tessler S. Ace inhibitor-induced angioedema in elderly African American females involving tracheostomy. J Natl Med Assoc 2010; 102: 529-530.

4. Seymour R A, Thomason J M, Nolan A. Angiotensin converting enzyme (ACE) inhibitors and their implications for the dental surgeon. Br Dent J 1997 . 183: 214-218

5. Wakefield Y S, Theaker E D, Pemberton M N. Angiotensin converting enzyme inhibitors and delayed onset, recurrent angioedema of the head and neck. Br Dent J 2008; 205: 553-556.

6. Gibbs C R, Lip G Y, Beevers D G. Angioedema due to 
ACE inhibitors: increased risk in patients of African origin. Br J Clin Pharmacol 1999; 48: 861-865

7. Kozel M M, Bossuyt P M, Mekkes J R, Bos J D. Laboratory tests and identified diagnoses in patients with physical and chronic urticaria and angioedema: a systematic review. J Am Acad Dermatol 2003; 48: 409-416.

8. Domenic A, Sica M D, Henry R, Black M D. ACE inhibitor-related Angioedema: can angiotensinreceptor blockers be safely used? Clin Hypertens 2002 4: 375-380.

9. Ugochukwu N. Diagnosis and management of angioedema with abdominal involvement: $\mathrm{A}$ gastroenterology perspective. World J Gastroentero 2010; 16: 4913-4921.

10. Michael W, Franz M. Angiotensin-converting enzyme inhibitors and angioedema: estimating the risk.
Hypertension 2008; 51: 1465-1467.

11. Peltekis G, Palaskas D, Samanidou M et al. Severe migratory angioedema due to ACE inhibitors use. Hippokratia: 2009; 13: 122-124.

12. Dean D E, Schultz D L, Powers R H. Asphyxia due to angiotensin converting enzyme (ACE) inhibitor mediated angioedema of the tongue during the treatment of hypertensive heart disease. J Forensic Sci 2001; 46: 1239-1243.

13. Ulmer J L, Garvey M J. Fatal angioedema associated with Lisinopril. Ann Pharmacother 1992; 26: $1245-1246$.

14. Stevenson H A, Steele J C, Field E A, Darrocj C J. Angioedema of the lips and tongue induced by angiotensin-converting enzyme inhibitor. A report of two cases. Prim Dent Care 2004; 11: 17-19.

15. Overlack A. ACE inhibitor-induced cough and bronchospasm. Incidence, mechanisms and management. Drug Safety 1996; 15: 72

16. National Institute of Clinical Excellence. Guideline 127: Clinical management of primary hypertension in adults. NICE, 2011.

17. Alexander G, Chiu M D, Edward J, Krowiak M D, Ziad E, Deeb M D. Angioedema associated with angiotensin II receptor antagonists: Challenging our knowledge of angioedema and its aetiology. The Laryngoscope 2001: 111: 1729-1173.

18. Hurst M, Empson M. Oral angioedema secondary to ACE inhibitors, a frequently overlooked association: case report and review. NZ Med J 2006; 119: U1930.

19. Juerg N, Massmo C, Marco C, Maggiore M D. Bradykinin-mediated angioedema. New Engl J Med 2002; 347: 621-622. 